Curley, L. J., Munro, J., Lages, M. , MacLean, R. and Murray, J. (2020) Assessing cognitive bias in forensic decisions: a review and outlook. Journal of Forensic Sciences, 63(2), pp. 354-360.

There may be differences between this version and the published version. You are advised to consult the publisher's version if you wish to cite from it.

This is the peer reviewed version of the following article:

Curley, L. J., Munro, J., Lages, M. , MacLean, R. and Murray, J. (2020) Assessing cognitive bias in forensic decisions: a review and outlook. Journal of Forensic Sciences, 63(2), pp. 354-360. (doi: 10.1111/1556$\underline{4029.14220}$ )

This article may be used for non-commercial purposes in accordance with Wiley Terms and Conditions for Self-Archiving.

http://eprints.gla.ac.uk/203759/

Deposited on: 20 December 2019

Enlighten - Research publications by members of the University of Glasgow http://eprints.gla.ac.uk/ 
Running Head: Is bias beneficial?

Title: Assessing cognitive bias in forensic decisions: A review and outlook.

Dr Lee J. Curley ${ }^{1 *}$, Dr James Munro², Dr Martin Lages ${ }^{3}$, Dr Rory MacLean², \& Associate Professor Jennifer Murray²

1. Faculty of Arts and Social Sciences, School of Psychology, the Open University, Milton Keynes, England.

2. School of Applied Sciences, Edinburgh Napier University, Edinburgh, Scotland.

3. College of Science and Engineering, The School of Psychology, The University of Glasgow, Glasgow, Scotland.

4. School of Health and Social Care, Edinburgh Napier University, Edinburgh, Scotland.

Word count (exc. tables/references):

*Requests for reprints should be addressed to Lee John Curley, Faculty of Arts and Social Sciences, School of Psychology, the Open University, Milton Keynes, England. (email: Lee.Curley@open.ac.uk 


\title{
Assessing cognitive bias in forensic decisions: A review and outlook.
}

\begin{abstract}
In recent years, a number of studies have demonstrated that forensic examiners can be biased by contextual information. However, concerns relating to methodological flaws and ecological validity attenuate how much the current body of knowledge can be applied to real-life operational settings. The current review takes a narrative approach to synthesising the literature across the forensic science. Further, the review considers both primary research on bias within forensic science and alternative theoretical perspectives surrounding contextual information and bias, to present an alternative view that bias does not always lead to error. The implications for future research are outlined, suggesting that studies on bias in forensic decisions should be conducted in collaboration between forensic scientists and cognitive psychologists. Only then rigorous and ecological valid experiments can be created that will be able to assess how bias influences forensic analysis and judgments in operationally valid settings.
\end{abstract}

Keywords: forensic science, forensic psychology, decision science, bias, forensic assessment, decision making. 
Evidence derived from traces of DNA is considered the gold standard of forensic science ${ }^{1}$ and is strongly weighted by jurors when reaching a verdict ${ }^{2}$. Within the courtroom, DNA evidence is viewed as an important tool when deciphering the guilt of a suspect. In addition to DNA evidence, different types of forensic evidence are typically analysed by forensic examiners to assist the court in its determination on substantive technical issues, which in turn may aid the jury in reaching a verdict (e.g., fingerprint analysis; ${ }^{3}$ ). Forensic evidence can be placed inside two broad categories: 1) pattern-matching techniques (non-scientific techniques that use terms such as 'match' and non-match; i.e., fingerprint analysis); and 2) DNA analysis (scientifically valid technique that uses probabilistic determinations; ${ }^{4}$ ). Recent research has highlighted that forensic evaluations of the first type may not be entirely objective ${ }^{5}$ and that subjectivity in pattern matching techniques may have contributed to miscarriages of justice, resulting in the payment of significant compensations ${ }^{6,7}$. Bias in forensic scientists is therefore a real cause for concern within the forensic science community ${ }^{8}$.

The National Academy of Sciences ${ }^{9}$ and the UK Forensic Science Regulator ${ }^{10}$ have suggested that forensic evaluations of pattern-matching techniques may be significantly affected by contextual bias. Previous research has shown that forensic examiners may fall prey to contextual and confirmation biases when ambiguous forensic information needs to be interpreted ${ }^{5,11}$. Contextual bias is the effect that extraneous information may have on the forensic examiners' decisions ${ }^{5}$, while confirmation bias occurs when an initial hypothesis influences how a decision maker interprets and/or searches for information, preferring information that supports their expectations ${ }^{12,13}$. Dror and Hampikian ${ }^{3}$ and Krane $^{14}$ suggested that contextual biases may also influence DNA evidence evaluations, as an example of the gold standard in forensic science. Their findings may have serious implications for both the prosecution and the defence due to the central importance and extensive use of DNA-related forensic evidence in the exoneration and incarceration of individuals ${ }^{15}$ and may call into 
question the validity of a significant number of verdicts, as some verdicts may have been rested upon biased forensic evaluations.

In this review we first discuss the empirical evidence of the effects that contextual information has on forensic scientists, followed by a discussion of the ecological validity and experimental rigour of these studies. We will argue that bias can also be beneficial. This will be based upon scientific evidence from the forensic science community and literature from decision science. Furthermore, we highlight potential issues with some of the current research on contextual bias in forensic scientists.

\section{Contextual Biases in Forensic Scientists.}

In recent years, research has highlighted that forensic science may not be as objective as commonly assumed by legal professionals and forensic scientists ${ }^{16}$. For instance, Dror et al. ${ }^{5}$ found that if ambiguous latent fingerprints (prints recovered from a crime scene that are invisible to the naked eye) were presented alongside contextual data, including emotionally resonant images and stories, then fingerprint examiners were increasingly likely to find a 'match' between prints found at the crime scene and a reference sample from the accused even if one did not exist. Dror, Wertheim, Fraser-Mackenzie and Walajtys ${ }^{17}$ further found that when fingerprint examiners were using an Automated Fingerprint Identification Systems (AFIS; a digital system that allows fingerprints to be stored, and searched for), latent fingerprints from the top of the AFIS list were much more likely to be matched incorrectly than fingerprints from the bottom of the AFIS list. Kukucka and $\operatorname{Kassin}^{18}$ found that handwriting comparisons were also influenced by the knowledge of confessions. Further, Cooper and Meterko ${ }^{19}$ conducted a systematic review and found that confirmation bias can influence the judgments of forensic scientists when contextual information is present. This shows that forensic examiners, just like 
other decision makers, are influenced by non-relevant information when making a decision. The inclusion of extra-legal factors in the decision processes can lead to more incorrect (or inaccurate) decisions being made which can have serious ramifications for decisions around the guilt and innocence of suspects in criminal cases.

Despite the apparent stability of these effects via replication across different forensic evaluation contexts (see Cooper and Meterko’s 19), some decision scientists have argued that biases and fallacies may be an artefact of experimental design and not necessarily the result of cognitive processing in a natural or typical environment ${ }^{19}$. Therefore, these experimental results may lack generalisability, and may be confined to psychologist's laboratories; this will be touched upon more in the next section. Nevertheless, high profile, real-life cases suggest that bias in forensic examiners may not just be confined to psychological studies. In 2004, the Federal Bureau of Investigation (FBI) mistakenly identified a Muslim male as being responsible for the Madrid train bombings ${ }^{16}$. The examiners had drawn the 'match' from a database that contained known prints. After this initial false positive, a number of other experts confirmed the false positive result $^{16}$. This real-life case demonstrated that forensic examiners who were provided with contextual information (i.e., surrounding the initial false positive) were more likely to confirm a match rather than disconfirm it. Dror et al. ${ }^{16}$ then used this real-life example in an experimental manipulation, with five fingerprint experts acting as participants in this study. Participants in this study were given prints from a crime scene, prints from a suspect, and were provided with contextual information. The context they were provided with was that the prints had been incorrectly identified by FBI experts when they were analysing evidence from the Madrid train bombing. Unknown to the fingerprint experts, they had previously identified the same prints as a match in another real-life case from their own case history. Four out of five participants changed their decision based on the context provided, thus demonstrating that forensic examiners can be easily influenced by contextual information. 
This confirmation bias extends to the evaluation and interpretation of different types of evidence, the most surprising of which is DNA evidence. Dror and Hampikian ${ }^{3}$ used a DNA mixture (biological material gained from more than one individual ${ }^{20}$ ) from a real case of gang rape to test whether or not ambiguous DNA evidence is also susceptible to bias. In this case, one of the individuals who had participated in the rape testified against another member (from which the target DNA was acquired) in exchange for a lower sentence and the forensic examiners concluded that the target DNA could not be excluded from the DNA mixture. In Dror and Hampikian's ${ }^{3}$ study, the same material was presented to 17 forensic examiners, but without any contextual information about the defendant/accused (i.e., the testimony from the assailant). From the 17 examiners, 12 decided that the target DNA could be excluded and four found that the DNA evidence was inconclusive. This study illustrates that the interpretation of ambiguous DNA evidence is not consistent, and that it can be influenced by contextual information.

The potential for subjectiveity when interpreting complex and ambiguous DNA mixtures was also shown in two further studies: DNA MIX05 and DNA MIX13 ${ }^{21}$. MIX05 was a study in which 69 forensic science laboratories evaluated and interpreted a two-person DNA mixture in four separate sexual assault mock trials ${ }^{21}$. The results of MIX05 showed that forensic scientists were more consistent when making judgements on major contributors (i.e., a complete, or almost complete DNA profile from a contributor is present) in two-person DNA mixtures when the genotypes of the contributors were not similar ${ }^{22}$, and that they were less consistent when making judgments on minor contributor genotypes ${ }^{22}$. In the MIX13 study, 108 forensic laboratories were presented with five complex mock crimes with up to four possible contributors to the DNA mixture ${ }^{21}$. The main finding of this study was that DNA mixture interpretations varied significantly across different forensic laboratories ${ }^{22}$. Both MIX05 and 
MIX13 showed that DNA mixtures are complex and ambiguous, which leaves this type of evidence open to influence from contextual biases ${ }^{3}$ and subjective reasoning.

The ambiguity of DNA evidence can also be influenced through factors such as environmental contamination (e.g., low template DNA - amplifying a limited amount of DNA so that it can be analysed ${ }^{23}$; and temperature at crime scene). Krane et al. ${ }^{24}$ suggested that DNA evidence is not always strong and that ambiguities allow contextual information to influence analysis and judgments. Forensic examiners typically use electropherograms when analysing DNA evidence. This equipment establishes stable positions on chromosomes (i.e., loci) and measures the presence of alleles (genetic variations ${ }^{25}$ ). The presence of an allele on a loci is represented on the user interface by a peak or bump, and no bump indicates that an allele is absent ${ }^{14}$. This method of analysis allows examiners to compare the DNA of a suspect with a DNA sample found at a crime scene. Sometimes, however, a number of factors (e.g., environmental contamination) can make it difficult to identify whether or not an allele is present on a loci (as peaks may be present but small), thus introducing noise in the analysis ${ }^{14}$. Krane ${ }^{14}$ proposed that this noise makes DNA evidence ambiguous, allowing contextual biases to affect the forensic examiners when analysing DNA evidence.

Thompson ${ }^{26}$ elaborated on the interaction between context and ambiguous evidence and suggested that false positives (e.g., the incarceration of innocent individuals) were most likely to occur due to subjective interpretations of ambiguous DNA evidence. Further, Thompson ${ }^{26}$ proposed that ambiguity arises from DNA results if the sample is small and/or degraded ${ }^{26}$. Limited DNA samples are also the samples which are least likely to be retested, because the samples may have been used-up in the initial testing. As a consequence, these limited samples are likely to be open to subjective interpretations, which may then influence how the jury perceives the defendant ${ }^{26}$. These subjective interpretations of DNA evidence may be the result of the forensic scientist having knowledge of the case and/or suspect ${ }^{27}$. Thompson (2011) 
further suggested that it is inappropriate for forensic scientists to consider contextual information, as their role is not trier of fact (i.e., they are not the jury) but to provide expert opinion on a piece of circumstantial evidence. This expert opinion should then be used alongside context by the trier of fact to assess the guilt of the defendant ${ }^{28}$, thus highlighting that context should be used by the jury but not forensic scientists as their roles differ in the legal system. In addition, Thompson's research ${ }^{26,28,29}$ therefore indicates that objective science has the potential for error as soon as subjective interpretations are involved.

A recent study by Kukucka, Kassin, Zapf and Dror ${ }^{30}$ showed that forensic experts also find it difficult to acknowledge bias in their own decision-making. Forensic decision makers were more likely to perceive bias in other forensic domains, less likely to perceive bias in their own domain, and least likely to acknowledge bias as a factor that might influence their own judgement ${ }^{30}$. This lack of awareness surrounding forensic bias shows that forensic scientists may be unconsciously integrating irrelevant contextual information alongside objective information from their forensic analysis when making matching decisions between a sample and a target. Furthermore, contextual and confirmation biases may be hidden from view in forensic science, as other biases (such as the over-confidence effect) may stop decision makers from being able to reflect on their own biases.

In summary, the aim of the current section was to highlight some issues in the literature on contextual information and its effects on forensic analysis and judgments. The current body of research has been primarily focussed on context and the negative effects it has on forensic scientists, despite research such as Dror et al. ${ }^{16}$ failing to measure the accuracy of the judgments made by forensic scientists. The next two sections will present alternative perspectives on the effects of context on forensic judgments. First, methodological limitations and concerns surrounding ecological validity will be presented, highlighting that it is to soon to establish how context influences forensic scientists in operationally valid settings. Second, 
an alternative perspective will be presented that will utilise commentary and research in the forensic science community and the theoretical background of bounded rationality from decision science. This alternative perspective will be that context (or bias) does not always lead to inaccurate decision making and may aid forensic scientists when they are interpreting forensic evidence.

\section{Experimental rigour and ecological validity in contextual bias investigations.}

Despite the amount of evidence suggesting that contextual information biases forensic scientists, there are two main issues with some of the literature that may attenuate how much the current body of knowledge can be applied to real world investigations: 1) experimental rigour (which will encompass for the purposes of this articles, all aspects of experimental investigations (i.e., sample size, recruitment styles, randomization processes); 2) ecological validity (as mentioned before). Each of these two issues will be addressed in turn.

First, as noted in Cooper and Meterko's ${ }^{19}$ systematic review, some of the research used in support of the negative effects that contextual biases have on forensic decisions have methodological flaws. Cooper and Meterko ${ }^{19}$ highlighted that sample sizes in articles investigating contextual bias can be limited, and thus attenuate the power and generalisability of the analyses. For example, in Dror et al.'s ${ }^{16}$ study on the effects of contextual information the sample size was five and the authors did not conduct inferential statistics, thus it is difficult to make generalisable conclusions from the study’s findings. Related to this, many studies in Cooper and Meterko's ${ }^{19}$ systematic review had not conducted statistical analysis correctly, as tests were sometimes conducted despite statistical assumptions being breached (e.g., chi-square analysis conducted despite expected frequencies being below five). In addition, the systematic review of Cooper and Meterko ${ }^{19}$ stated: “...none of the studies provided information about the 
randomization procedures, as is recommended for randomized clinical trials” (p.42), thus highlighting that these studies are not examples of true experiments. In a proper experimental design participants are randomly allocated to conditions to decrease the effects of individual differences in performance and systematic error, thus research investigations that do not randomly allocate participants cannot rule out that their results may have been influenced by individual differences and error. Similarly, Dror et al.'s ${ }^{16}$ study lacked a control group, making inferences about the effects of contextual bias difficult. A final methodological flaw that was highlighted by Cooper and Meterko’s ${ }^{19}$ systematic review was that studies investigating bias in forensic science differed in their approach to how naive the participants were about the experimental designs and hypotheses, thus making it difficult to compare across studies, and differentiate when the effects of bias were down to the presentation of contextual information or when it was simply caused by demand characteristics.

A second important question surrounding the experimental literature on contextual biases is, to what extent do laboratory experiments that induce bias translate to typical forensic decisionmaking? This question is difficult to answer, as some research tested forensic scientists and the effects of context in the normal working environments of forensic scientists (e.g., Dror et al. ${ }^{16}$ ), whereas other studies have been conducted using small student-based samples in artificial environments (e.g., Dror et al. ${ }^{5}$ ). These different methods of investigating forensic bias relate to what Towler et al. ${ }^{\text {51change this }}$ called operational and perceptual accuracy. Operational accuracy is tested when the decision making of forensic scientists is tested in studies that represent reallife casework in operationally valid settings. In contrast, perceptual accuracy is measured in an experimental setting that is representative of real-life case work, but is not operationally valid, as commonly used tools and scales might not be available ${ }^{51}$ change, white Philips et al. 2015 . Most research to date has focussed primarily on the latter over the former, meaning that the effects 
of bias in real-life forensic decisions is still not fully understood ${ }^{51}$, and consequently needs more invesitation.

In summary, despite the explosion of research relating to bias and forensic science, some of the previous research has been lacking in experimental rigour and ecological validity. Therefore, before recommendations and conclusions can be drawn about the effects of contextual information on forensic scientists in their daily decisions, future studies are needed which test the effects of bias on operational accuracy in ecologically valid settings using proper experimental designs. Because of these methodological limitations, the next section of this review will discuss an alternative account of the effects of contextual information on forensic judgments.

\section{The Good, the Bad and the Biased: Do biases equal error?}

Although contextual information might lead to biased decisions, this does not necessarily mean that forensic examiners who are presented with contextual information will make incorrect decisions. The aim of the current section, therefore, is to present commentary, theory and research supporting the idea that context does not necessarily lead to inaccurate decision making and that sometimes context (or bias) may promote accurate decision making.

Searston, Tangen and $\mathrm{Eva}^{45}$ conducted three experiments to investigate whether or not contextual information had an effect on the accuracy of forensic judgments when analysing latent fingerprints, or whether it simply caused a response bias (or beta as it will now be referred to). In their second experiment, Searston et al. ${ }^{45}$ found that the decisions forensic examiners made were biased by familiarity. Their experiment consisted of a learning phase followed by a test phase. In the learning phase, participants were given contextual information surrounding a case, then asked to make a decision (match vs. non-match). Participants were provided with 
feedback in this initial stage, allowing participants to learn a false (i.e., experimentally induced) association between the decision match and the given context. Familiarity of context was associated with a decrease in accuracy, suggesting that familiarity may bias decisions and can lead to inaccurate decisions.

In Searston et al.'s ${ }^{45}$ third experiment, they investigated whether similar fingerprints from previous mock trials could bias judgements in subsequent trials and thus change the accuracy of the decision maker. In this experiment, no context was provided, and participants were given the same latent fingerprints twice, once in the learning phase (with feedback) and once in the test phase (with no feedback). Participants performed worse in the test phase in comparison to the learning phase. This suggests that familiarity with fingerprints might bias individuals to make a decision consistent with the decision they made on a previous case with a similar looking fingerprint, thus biasing the forensic examiner and potentially leading to less accurate decisions.

Further, Searston et al.' ${ }^{45}$ second and third experiment showed, in line with previous research, that contextual information may cause individuals to make a biased or incorrect decision. However, this bias does not originate from contextual information regarding a case or a suspect, rather this bias originates with the experience of the forensic scientists regarding familiar pieces of evidence ${ }^{47}$. In other words, the more experience you have of evaluating fingerprints, the more fingerprints you have seen, which may leave you more vulnerable to Searston et al.'s ${ }^{45}$ familiarity effect. Goldstein and Gigerenzer ${ }^{41}$ suggested that the experience (or expertise) an individual has with a particular environment can influence the accuracy of decision making, with no or too much experience having a negative effect on decision making. This is because if you have no knowledge of an environment you will not be able to reliably discriminate between two outcomes, whereas if you have too much information available to you, the complexity of the decision increases which can make it more difficult to choose the correct 
option. They suggest that individuals with intermediate knowledge of an environment can make the most accurate decisions, as they use just enough information to discriminate between the options but not too many cues to be confused and overwhelmed by the information ${ }^{41}$. However, it should be mentioned that in Goldstein and Gigerenzer's ${ }^{41}$ research the participants were lay people who were asked to make decisions surrounding particular aspects of a city and which city was greater/lesser in regard to these aspects (e.g. the most populous city out of the two cities presented). Therefore, Goldstein and Gigerenzer's ${ }^{41}$ work does not directly apply to forensic science, but their theoretical framework surrounding rationality that is bounded by the environment may help to aid why expertise can affect decisions in paradoxical ways.

Dror $^{47}$ offered an alternative explanation for why expertise may have a negative influence on forensic examiners. He suggested that expertise allows forensic experts to create schemas (mental models) of decisions and their associated environments, which can then create rigid thinking processes, thus limiting creativity and biasing the decision outcome to their previous experience. Similar to Searston et al. ${ }^{45}$, Dror ${ }^{47}$ suggested that familiarity with contextual and non-contextual (i.e., forensic evidence) information may cause an error, rather than the contextual information itself. Expertise may lead to forensic decision makers utilising top down processes more than bottom up processes, which may cause the decision maker to rely on familiar information more than novel information. This may have some negative outcomes: 1) the forensic decision maker may overestimate the importance of familiar information; 2) the forensic decision maker may underestimate the importance of novel information ${ }^{31,47}$. These top-down processes, however, will typically lead to more accurate decision making in simple and regular decisions, but may lead to error in complex and irregular decisions (i.e., when ambiguous information is present ${ }^{16,31,47}$. 
However, Searston et al.'s ${ }^{45}$ first experiment highlighted that context does not always lead to inaccurate decisions, suggesting that some contextual information may randomly affect accuracy $^{48}$. they found that case severity (high vs. low) had an impact on the beta of forensic examiners but did not influence their overall accuracy. In other words, forensic examiners were more likely to "match" a sample with a target in cases with high severity than in cases with low severity, but this tendency was not indicative of whether a correct match was established or not. Therefore, it can be said that context is not always a significant contributor to erroneous judgement.

Langenburg, Champod and Wertheim ${ }^{49}$ found similar effects when comparing effect of context and expertise on errors and beta (decision outcome matched the contextual information) for matching unknown fingerprints with a fingerprint exemplar ${ }^{49}$. They found that novices were influenced more by bias than experts, and that the novice group made significantly more errors (number of errors $=46$ ) than the expert group (number of errors $=4$ ). This is in contrast to the findings discussed earlier, demonstrating that expertise effects are complex, and are likely to be affected by different contexts and types of decisions. Further, this highlights that the presence of biasing contextual information does not always lead to inaccurate decision making in expert forensic scientists.

Kerstholt et al. ${ }^{50}$ investigated whether contextual information had an effect when assessing whether a bullet was fired from a certain firearm or not in two investigations. The first investigation found that contextual information had no effect on the decision outcome. In the second investigation, the researchers compared the decision of a first forensic scientist (the person who conducted the initial assessment) with the decision made by a second forensic scientist (the person who conducted the second assessment) in real-life cases. It was expected that the first examiner would have been more extreme as they would have had more contextual information available to them. The second examiner, however, gave a more biased answer and 
were more likely than the first examiner to suggest that the firearm being assessed did fire the bullets being analysed than the first examiner. In addition, Kerstholt, Paashious, Sjerps ${ }^{\text {addnumber }}$ found in a separate study that contextual information did not influence assessments in shoe print examinations. In summary, the studies by Searston et al. ${ }^{45}$, Langenburg et al. ${ }^{49}$, and Kerstholt et al. ${ }^{50}$ highlight that bias does not necessarily lead to more inaccurate decision making. Therefore, bias should not always be seen as negative, and more research is needed to consider whether bias can be beneficial and when bias has no effect.

As mentioned before, some researchers have suggested that heuristics (cognitive short-cuts) and bias can lead to more accurate decision making. For instance, Goldstein and Gigerenzer ${ }^{41}$ theorised about, and have since tested, fast and frugal heuristics that allow decisions to be made both quickly and accurately. They claimed that contextual/environmental information may be beneficial when making decisions as it scaffolds our limited cognitive structures (i.e., heuristics), thus bounding rationality to the environment. Further, they suggested that our minds have evolved to adapt to our environment, and that we have a toolbox of heuristics that allow us to access the best information quickly, rather than integrating all the information, to make a decision. These heuristics vary according to their 'satisficing rule' (from using one cue to a number of cues), but in each of the heuristics, it is the best piece of information (i.e., a piece of information that allows two outcomes to be discriminated against) that allows us to make good decisions quickly. In other words, information that biases decision processes may promote accurate and quick decision making. Goldstein and Gigerenzer ${ }^{41}$ also suggested that through interaction with the environment, we learn which pieces of information are most likely to be associated with the correct outcome, thus allowing fast and frugal decisions to be made. Furthermore, the fast and frugal paradigm has contributed two important findings to psychological research: 1) that the utilisation of environmental/contextual information is crucial to make accurate decisions; 2) bias may be beneficial to decision making. 
Within an ecologically valid forensic setting it is indeed possible that some contextual information may be actually beneficial. For instance, Towler et al. ${ }^{51}$ stated that: "Unlike most experiments, the ratio of targets to non-targets in forensic contexts is almost certainly not 50:50" (p.204). In real-life forensic examinations, there will be a higher chance of samples being a match than a non-match ${ }^{51}$. Therefore, contextual information that biases forensic scientists in favour of the prosecution may actually induce correct decision making, even though the practice of utilising contextual information may be ethically questionable and not justifiable legally ${ }^{28}$; this will be discussed more later with the 'criminalist paradox'. In addition, Rudin and Inman $^{52}$ suggested that if a forensic scientist is unaware of the context surrounding the case, they may conduct tests that are either worthless or dangerous. They propose that context is needed so that forensic scientists know which question to answer and this then informs the tests that are conducted and the evidence which is collected. They propose that contextual ignorance may have more of a negative effect on forensic evaluations than contextual bias does. Furthermore, in real-life laboratories contextual information and bias may aid forensic scientists to evaluate forensic evidence.

Whitman and Koppl ${ }^{42}$ have suggested that the utilisation of context in forensic examiners may even be a rational endeavour. This is because the decision that is reached will be informed by both prior beliefs surrounding the accused's guilt (which may have arisen from information from the police, and the subjective weight they place on incarcerating the guilty vs. the innocent) and evidence; thus, mirroring the updating of priors in Bayesian statistics. Further, Evett, Jackson, and Lambert ${ }^{43}$ proposed that DNA caseworkers use the Case Assessment and Interpretation (CAI) model. This model requires forensic scientists to seek as much contextual information as possible from the investigator. Evett et al. ${ }^{43}$ also suggested that the utilisation of contextual information allows examiners to update their beliefs in a manner that mirrors a Bayesian process of updating, thus hinting that contextual information may allow forensic 
scientists to perform more rationally. Furthermore, forensic examiners who use contextual information may doing so in a manner that is associated with rational decision making.

However, despite the potential positive effect of context when analysing forensic evidence, it may be legally/ethically unsuitable to use contextual information as it may lead to a 'criminalist paradox ${ }^{28}$ (p.130). Thompson ${ }^{28}$ stated that contextual information may aid forensic scientists to make the correct decision, as a forensic scientist may be aided in finding a correct match between fingerprints when they have learned that the suspect, from whom the latent fingerprints have been collected, has confessed. The problem arises when jurors have to decide on an appropriate verdict. According to standard Bayesian modelling, jurors should integrate each piece of information gained from evidence surrounding the guilt of the defendant independently of one another before arriving at a decision ${ }^{28,}{ }^{37}$. However, if jurors are integrating confession evidence and forensic evidence independent of one another, when really each piece of evidence is correlated, then jurors will think that the likelihood of the suspect being guilty is much higher than it actually is ${ }^{28}$.

For example, in a case which led to Josiah Sutton being incorrectly convicted of rape, the interpretations of a DNA mixture were influenced by the identification of the victim ${ }^{29}$. It was also reported that the victim's testimony in court may have been influenced by the knowledge of the DNA results. The case against Josiah Sutton, according to Bayesian modelling, was weak as each piece of evidence was dependent on the other but was perceived as strong by the jury as they expected that each piece of evidence originated from independent sources ${ }^{29}$. Furthermore, contextual information may aid individual forensic analysts to make a correct judgement but may have serious negative implications surrounding the outcome of a trial by jury. 
Nevertheless, despite the 'criminalist paradox' academics and practitioners in the forensic science community have commented on the utility of contextual information. For instance, Budowle et al. ${ }^{48}$ suggested that some contextual information aids the decision-making process of forensic examiners to prioritise the most meaningful examples when there is a plethora of evidence samples. Therefore, similar to Goldstein and Gigerenzer's ${ }^{41}$ fast and frugal heuristics, information from the environment (i.e., context of case) may aid forensic decision makers to process and analyse the most useful samples, thus allowing the forensic examiner to make an accurate decision despite using limited resources (both cognitive and evidentiary) ${ }^{48}$. Kruse $\mathrm{e}^{55}$ advocated that contextual information surrounding a case (i.e., when the case occurred), familiarity with previous cases (experience of analysing similar types of materials), and teachings from colleagues allow forensic scientists to better evaluate forensic evidence. Further, Elaad ${ }^{57}$ proposed that contextual information may have a positive relationship with the accuracy of a judgement and does not always cause an inaccurate judgement to be reached. Butt $^{56}$ even suggested that the negative effects of contextual bias are overestimated in psychological literature, and that contextual information may influence forensic scientists in a positive manner. In summary, the effects that context have on the judgements and analysis of forensic scientists may not always be negative.

\section{Conclusion}

The current body of knowledge surrounding the effects of context on forensic judgments and analysis has mostly suggested that contextual information may cause bias in forensic examiners when analysing different types of forensic evidence (e.g., fingerprint analysis, handwriting, and DNA mixture analysis). It has also been suggested that the biases created by contextual information may have a negative influence on the accuracy of the decisions made by forensic 
scientists. However, because of methodological flaws (inaccurate inferential statistics; small sample sizes; no information on the allocation of groups) and problems with the ecological validity of previous experiments, these results may not generalise to the real-life judgments made by forensic scientists. Therefore, the current review aimed to present an alternative perspective which has been commented on by forensic scientists, researched by academics and is supported by the theorical framework of bounded rationality. This alternative perspective is that context (or bias) may not always influence forensic decisions in a negative manner, and that context may aid forensic scientists when making decisions regarding forensic evidence. To test this alternative perspective, we recommend that forensic scientists and cognitive psychologists collaborate to create rigorous and ecologically valid experiments that will be able test the effects that context and bias have on forensic scientists in operationally valid settings. 


\section{References}

1. Lynch M. God's signature: DNA profiling, the new gold standard in forensic science. Endeavour 2003; 27(2): 93-97.

2. Briody M. The effects of DNA evidence on homicide cases in court. Australian \& New Zealand Journal of Criminology 2004; 37(2): 231-252.

3. Dror I, Hampikian G. Subjectivity and bias in forensic DNA mixture interpretation. Science and Justice 2011; 51: 204-208.

4. Richmond, K. The NAS/NRC Report: Global Perspectives, Ongoing Challenges [Blog $\begin{array}{lllll}\text { post]; } & 2019 & \text { April } & 15 . & \text { Available }\end{array}$ https://lawandscienceweb.wordpress.com/2019/04/15/the-nas-nrc-report-globalperspectives-ongoing-challenges/

5. Dror IE, Peron AE, Hind SL, Charlton D. When emotions get the better of us: the effect of contextual top-down processing on matching fingerprints. Applied Cognitive Psychology: The Official Journal of the Society for Applied Research in Memory and Cognition 2005; 19(6): 799-809.

6. Garrett BL, Neufeld PJ. Invalid forensic science testimony and wrongful convictions. Virginia Law Review 2009; 95(1): 1-97.

7. Smit NM, Morgan RM, Lagnado DA. A systematic analysis of misleading evidence in unsafe rulings in England and Wales. Science \& Justice 2018; 58(2):128-137.

8. Science and Technology Select Committee. Forensic science and the criminal justice system: a blueprint for change. House of Lords (HL); 2019 May 1st. Report no.:3. HL paper: 333.

9. National Academy of Sciences. Strengthening Forensic Science in the United States: A Path Forward. Washington, DC: National Academies Press, 2009.

10. Tully G. Annual Report. (2015). Forensic Science Regulator; 2015 December 4. 
11. Kaplan MF, Miller LE. Reducing the effects of juror bias. Journal of Personality and Social Psychology 1978; 36(12):1443-1455.

12. Findley KA, Scott MS. The multiple dimensions of tunnel vision in criminal cases. Wisconsin Law Review 2006; 2: 1-107.

13. Nickerson RS. Confirmation bias: A ubiquitous phenomenon in many guises. Review of general psychology 1998; 2(2): 175-220.

14. Krane. D. E. Exploring bias in forensic DNA profiling [Video file]; 2015 January 12th. Available from: https://www.youtube.com/watch?v=tpPkmDeS3Dg

15. Kassin SM, Dror IE, Kukucka J. The forensic confirmation bias: Problems, perspectives, and proposed solutions. Journal of Applied Research in Memory and Cognition 2013; 2(1): 42-52.

16. Dror IE, Charlton D, Péron AE. Contextual information renders experts vulnerable to making erroneous identifications. Forensic science international 2006; 156(1):74-78.

17. Dror IE, Wertheim K, Fraser-Mackenzie P, Walajtys J. The impact of humantechnology cooperation and distributed cognition in forensic science: biasing effects of AFIS contextual information on human experts. Journal of forensic sciences 2012; 57(2): 343-352.

18. Kukucka J, Kassin SM. Do confessions taint perceptions of handwriting evidence? An empirical test of the forensic confirmation bias. Law and Human Behavior 2014; 38(3): 256-270.

19. Cooper GS, Meterko V. Cognitive bias research in forensic science: A systematic review. Forensic science international 2019; 297: 35-46.

20. Gigerenzer G, Hoffrage U, Kleinbölting H. Probabilistic mental models: A Brunswikian theory of confidence. Psychological review (1991); 98(4): 506-528. 
21. Bieber FR, Buckleton JS, Budowle B, Butler, JM, Coble MD. Evaluation of forensic DNA mixture evidence: protocol for evaluation, interpretation, and statistical calculations using the combined probability of inclusion. BMC genetics 2016; 17(1): $1-15$.

22. Augenstein S. NIST Publishes Landmark MIX13 DNA Study. Forensic Magazine 2018 February $2^{\text {nd }}$. Available from: https://www.forensicmag.com/news/2018/08/nistpublishes-landmark-mix13-dna-study

23. Butler M, Kline MC, Coble MD. NIST interlaboratory studies involving DNA mixtures (MIX05 and MIX13): Variation observed and lessons learned. Forensic Science International: Genetics 2018; 37: 81-94.

24. The Forensic Institute [internet]. Low copy number or low template DNA analysis; c2019 [cited 2019 August 20. Available from: http://www.theforensicinstitute.com/news-articles/research/dna/low-template-or-lowcopy-number

25. Krane DE, Ford S, Gilder JR, Inman K, Jamieson A, Koppl R et al. Sequential unmasking: a means of minimizing observer effects in forensic DNA interpretation. Journal of Forensic Sciences 2008; 53(4): 1006-1007.

26. Gyllensten UB, Erlich HA. Generation of single-stranded DNA by the polymerase chain reaction and its application to direct sequencing of the HLA-DQA locus. Proceedings of the National Academy of Sciences 1988; 85(20): 7652-7656.

27. Thompson WC. Accepting lower standards: the national research council's second report on forensic DNA evidence. Jurimetrics 1997; 37 (4): 405-424.

28. Cole SA, Thompson WC. Forensic science and wrongful convictions. In: Huff RC, Killias M, editors. Wrongful convictions and miscarriages of justice: Causes and 
remedies in North American and European criminal justice systems. New York, NY: Routledge, 2013;122-146.

29. Thompson WC. What role should investigative facts play in the evaluation of scientific evidence?. Australian Journal of Forensic Sciences 2011; 43(2-3): 123-134.

30. Thompson WC. Beyond bad apples: Analyzing the role of forensic science in wrongful convictions. Sw. UL Rev. 2008; 37: 1027-1050.

31. Kukucka J, Kassin SM, Zapf PA, Dror IE. Cognitive bias and blindness: A global survey of forensic science examiners. Journal of Applied Research in Memory and Cognition 2017, 6(4), 452-459.

32. Tversky A, Kahneman D. Judgment under uncertainty: Heuristics and biases. Science, New Series 1974; 185 (9): 1124-1131.

33. Pronin E, Gilovich T, Ross L. Objectivity in the eye of the beholder: divergent perceptions of bias in self versus others. Psychological review 2004;111(3):781-799.

34. Lecci L, Myers B. Individual differences in attitudes relevant to juror decision making: Development and validation of the pretrial juror attitude questionnaire (PJAQ) 1. Journal of Applied Social Psychology 2008; 38(8): 2010-2038.

35. Guthrie C, Rachlinski JJ, Wistrich AJ. Judging by heuristic-cognitive illusions in judicial decision making. Judicature 2002; 86 (1): 44-50.

36. Smalarz L, Madon S, Yang Y, Guyll M, Buck S. The perfect match: Do criminal stereotypes bias forensic evidence analysis?. Law and Human Behavior 2016; 40(4): 110.

37. Kahneman D. Thinking, fast and slow. International ed. London, U.K.: Penguin book, 2011.

38. Curley LJ, MacLean R, Murray J, Laybourn P. Decision Science: A New Hope. Psychological reports 2018; 121 (5): 1-23. 
39. Evans JSB. In two minds: dual-process accounts of reasoning. Trends in cognitive sciences 2003; 7(10): 454-459.

40. Zhang KZ, Zhao SJ, Cheung CM, Lee MK. Examining the influence of online reviews on consumers' decision-making: A heuristic-systematic model. Decision Support Systems 2014; 67:78-89.

41. Chan J, Wang J. Hiring biases in online labor markets: The case of gender stereotyping. Proceedings of the ICIS; 2014 December 14-17; Auckland, NZ.

42. Goldstein DG, Gigerenzer G. (2002). Models of ecological rationality: the recognition heuristic. Psychological review 2002; 109(1): 75-90.

43. Whitman G, Koppl R. Rational bias in forensic science. Law, probability and risk 2010; 9(1): 69-90.

44. Evett IW, Jackson G, Lambert JA. More on the hierarchy of propositions: exploring the distinction between explanations and propositions. Science \& justice: journal of the Forensic Science Society 2000; 40(1): 3-10.

45. Dror IE, Thompson WC, Meissner CA, Kornfield I, Krane D, Saks M et al. Letter to the editor-context management toolbox: a linear sequential unmasking (LSU) approach for minimizing cognitive bias in forensic decision making. Journal of Forensic Sciences 2015; 60 (4): 1-2.

46. Searston RA, Tangen JM, Eva KW. Putting bias into context: The role of familiarity in identification. Law and Human Behavior 2016; 40(1): 50-64.

47. Risinger DM, Saks MJ, Thompson WC, Rosenthal R. The Daubert/Kumho implications of observer effects in forensic science: Hidden problems of expectation and suggestion. Californian Law Review 2002; 90 (1): 1-56. 
48. Dror IE. The paradox of human expertise: why experts get it wrong. In: Kapur N, Pascual-Leone A, and Ramachandran VS, editors. The paradoxical brain. Cambridge, U.K.: Cambridge University Press, 2018; 177-188.

49. Budowle B, Bottrell M C, Bunch SG, Fram R, Harrison D, Meagher S et al. A perspective on errors, bias, and interpretation in the forensic sciences and direction for continuing advancement. Journal of Forensic Sciences 2009; 54(4): 798-809.

50. Langenburg G, Champod C, Wertheim P. Testing for potential contextual bias effects during the verification stage of the ACE-V methodology when conducting fingerprint comparisons. Journal of Forensic Sciences 2009; 54(3): 571-582.

51. Kerstholt J, Eikelboom A, Dijkman T, Stoel R, Hermsen R, van Leuven, B. Does suggestive information cause a confirmation bias in bullet comparisons?. Forensic science international 2010; 198(1-3): 138-142.

52. Towler A, White D, Ballantyne K, Searston RA, Martire KA, Kemp RI. Are forensic scientists experts?. Journal of Applied Research in Memory and Cognition 2018; 7(2):199-208.

53. Rudin N, \& Inman K. How much should an analyst know? CACNews 1997. Available from: http://www.forensicdna.com/assets/how-much-should-an-analyst-know_.pdf

54. Thompson MB, McCarthy DJ, Tangen JM. Human matching performance of genuine crime scene latent fingerprints. Law and human behavior 2014; 38(1):1-10.

55. Verkaik R. 'It's better that 10 guilty men go free than one innocent man be wrongly convicted'. The Independent 2008 September 27th. Retrieved from: https://www.independent.co.uk/news/uk/home-news/its-better-that-10-guilty-men-gofree-than-one-innocent-man-be-wrongly-convicted-944059.html

56. Kruse C. The Bayesian approach to forensic evidence: Evaluating, communicating, and distributing responsibility. Social Studies of Science 2013; 43(5): 657-680. 
57. Butt L. The forensic confirmation bias: Problems, perspectives, and proposed solutions: Commentary by a forensic examiner. Journal of Applied Research in Memory \& Cognition 2013; 2: 59 - 60 .

58. Elaad E. (2013). Psychological contamination in forensic decisions. Research in Mmeory and Cognition 2013; 2 (1): 61-62.

59. Charlton D. Standards to avoid bias in fingerprint examination: Are such standards doomed to be based on fiscal expediency? Journal of Applied Research in Memory \& Cognition 2013; 2: 71-72.

60. Dror I, Cole S. The Vision in blind justice: Expert perception, judgement, and visual cognition in forensic pattern recognition. Psychonomic Bulletin \& Review 2010;17:161-167.

61. Campbell A. The fingerprint enquiry report, 2011 December $14^{\text {th }}$. Available from: https://keithborer.co.uk/news/scottish-fingerprint-inquiry-report-published-14thdecember-2011

62. Cole S. Implementing Counter-measures against confirmation bias in forensic science. Journal of Applied research in Memory and Cognition 2013; 21 (1):46-47.

63. Dror IE, Kassin SM, Kukucka. New application of psychology to law: Improving forensic evidence and expert witness contributions. Journal of Applied Research in Memory and Cognition 2013; 2(1): 78-81.

Add number too: White D, Phillips PJ, Hahn CA, Hill M, O'Toole AJ. (2015). Perceptual expertise in forensic facial image comparison. Proceedings of the Royal Society B: Biological Sciences 2015; 282(1814): 1-8.

Kerstholt JH, Paashuis R, Sjerps M. Shoe print examinations: effects of expectation, complexity and experience. Forensic science international 2007; 165(1): 30-34. 